\title{
Cuidados paliativos e o ensino médico mediado por tecnologias: avaliação da aquisição de competências
}

\section{Palliative care and technology-mediated learning: assessment of the acquisition of competencies}

\author{
Amanda Carla de Souza Kanashiro' (1) | amandaakanashiro@gmail.com \\ Rebeca Isabela Ciardulo Marques Grandini' (1) rebeca18.2ufpr@gmail.com \\ Úrsula Bueno do Prado Guirro' (1) ursula@ufpr.br
}

\begin{abstract}
RESUMO
Introdução: $\mathrm{O}$ ensino de cuidados paliativos (CP) é essencial na educação médica. Devido à pandemia da Sars-Cov-2, foi necessário adaptar o ensino presencial para o mediado por tecnologias, e não se sabia se o método era capaz de proporcionar a aquisição de competências aos estudantes.

Objetivo: Este estudo teve como objetivo avaliar se ocorreu a aquisição de competências em CP entre os estudantes de Medicina matriculados em uma disciplina de CP mediada por tecnologias.

Método: Estudantes de Medicina matriculados na disciplina de CP mediada por tecnologias foram convidados para participar do estudo. Ao longo de sete semanas, abordaram-se as temáticas essenciais dos CP. Houve atividades síncronas e assíncronas, estudo dirigido, problematização e simulação sem pacientes reais. Utilizou-se o questionário PalliComp antes do início das atividades didáticas e ao final para avaliar a aquisição de competências. Os dados obtidos foram submetidos à análise estatística.

Resultado: Dos 45 estudantes matriculados, 37 responderam ao questionário PalliComp antes da disciplina e 32 no final. A amostra foi constituída de $68,9 \%$ de mulheres e $31,1 \%$ de homens, com idade média de $23,9 \pm 3,5$ anos. O escore geral de competências elevou-se de $63,9 \pm 14,7$ para $74,9 \pm 14,6(0,001)$ em uma escala que variava de 0 a 100. A elevação estatisticamente significativa ocorreu nas competências relacionadas ao conceito de $\mathrm{CP}(<0,001)$, à abordagem de sintomas físicos $(0,004)$ e psicoemocionais $(<0,001)$, à família $(0,03)$ e à tomada de decisão ética $(0,05)$. Não mostraram diferença as competências de abordagens social $(0,07)$ e espiritual $(0,13)$, trabalho em equipe $(0,67)$, comunicação $(1,00)$ e autodesenvolvimento $(0,13)$.
\end{abstract}

Conclusão: Houve aquisição geral de competências em CP entre estudantes de Medicina, e a estratégia de ensino em uma disciplina específica mediada por tecnologias se mostrou válida.

Palavras-chave: Cuidados Paliativos; Educação a Distância; Educação Baseada em Competências; Educação Médica.

\section{ABSTRACT}

Introduction: Teaching palliative care is essential in medical education. The pandemic forced the adaptation of classroom learning to technologymediated learning, and it is not known whether the method would adequately provide students with the acquisition of competencies.

Objective: To assess whether palliative care competencies are acquired by medical students enrolled in a technology-mediated palliative care discipline.

Methods: Medical students enrolled in the technology-mediated palliative care discipline were invited to participate in the study. Over a seven-week period, the essential themes of palliative care were addressed. There were synchronous and asynchronous activities, directed study, problematization and simulation without real patients. The PalliComp questionnaire was used prior to the beginning of the teaching activities and at the end to assess the acquisition of competencies. The data obtained were submitted to statistical analysis.

Results: Of the 45 students enrolled, 37 answered the PalliComp questionnaire before the course and 32 at the end. The sample consisted of $68.9 \%$ women and $31.1 \%$ men, with a mean age of $23.9 \pm 3.5$ years. The overall competency score increased from $63.9 \pm 14.7$ to $74.9 \pm 14.6$ (0.001) on a scale ranging from 0 to 100. Statistically significant increases were found in the competencies related to the concept of palliative care $(<0.001)$, approach to physical symptoms (0.004), psycho-emotional $(<0.001)$, family (0.03) and ethical decision-making (0.05). No difference was found in the competencies of social approach (0.07), spiritual (0.13), teamwork (0.67), communication (1.00) and self-development (0.13). Conclusion: A general acquisition of competencies in palliative care was verified among medical students and the technology-mediated learning strategy in a specific discipline proved to be valid.

Keywords: Palliative Care; Remote Learning; Competency-Based Education; Medical Education.

${ }^{1}$ Universidade Federal do Paraná, Curitiba, Paraná, Brasil.

Editora-chefe: Rosiane Viana Zuza Diniz.

Editor associado: Pedro Tadao Hamamoto Filho.

Recebido em 11/06/21; Aceito em 24/08/21.

Avaliado pelo processo de double blind review. 


\section{INTRODUÇÃO}

Os Cuidados Paliativos (CP), de acordo com a Organização Mundial da Saúde, referem-se ao conjunto de terapias assistenciais em saúde promovidas por uma equipe multidisciplinar, com o objetivo de melhorar a qualidade de vida dos pacientes (adultos e crianças) e de seus familiares, diante de doenças que ameaçam a continuidade da vida. Por meio dos $\mathrm{CP}$, é possível prevenir e aliviar o sofrimento, identificar precocemente as necessidades do paciente, realizar uma avaliação impecável e tratar da dor e dos demais sintomas físicos, sociais, psicológicos e espirituais'. São evidentes a importância desses cuidados e a magnitude de pacientes em fase terminal que podem ter o sofrimento reduzido com a assistência paliativa estruturada diante de doenças incuráveis.

Os saberes envolvidos nos CP podem ser elencados em três níveis. $O$ primeiro consiste na abordagem paliativa, que é o conhecimento que todo profissional de saúde, incluindo o médico, deveria receber na graduação e, portanto, poderia ofertar aos pacientes portadores de doenças incuráveis na trajetória de enfrentamento do adoecimento, quando não existem sintomas complexos. O segundo nível é denominado cuidados paliativos gerais, que consistem nos saberes dos profissionais que lidam frequentemente com pessoas com necessidades paliativas, como a oncologia e a terapia intensiva, mas não são especialistas em $\mathrm{CP}$, ou seja, incluem a atenção em CP com a especialidade primária. No terceiro nível, estão os cuidados paliativos especializados, que tratam do conjunto de abordagens ofertadas pelos especialistas em CP e atendem pacientes com demandas de maior complexidade ${ }^{2,3}$.

Dessa maneira, compreende-se que as competências envolvidas na abordagem paliativa deveriam ser conhecidas por todos os médicos generalistas, e, por esse motivo, o ensino deveria ser ofertado na graduação ${ }^{4}$. Trata-se de temática básica, não especializada, em que se abordam a dor e outros sintomas desconfortáveis, a comunicação de más notícias, o acolhimento e apoio na organização familiar, o trabalho em equipe multidisciplinar, as questões relacionadas com a bioética da terminalidade, entre outros aspectos ${ }^{2,3}$.

Todavia, de acordo com a Academia Nacional de Cuidados Paliativos, apenas $14 \%$ dos cursos de Medicina do Brasil ofertam disciplina direcionada para os CP na grade curricular. No caso dos CP, não cabe a expressão "bom senso", pois eles exigem aprendizado, assim como outras áreas da medicina. Se forem ofertados de maneira estruturada, haverá melhora da qualidade de vida dos pacientes adoecidos, diminuição dos sintomas desconfortáveis e redução de custos desnecessários ${ }^{5,6}$.

Assim, há um problema a ser abordado na formação médica: a capacitação dos futuros médicos para cuidar de pessoas portadoras de sofrimentos relacionados com a terminalidade da vida. O ensino não se dá apenas no currículo formal, mas também em todas as aulas, atividades e vivências do estudante de Medicina relacionadas com pacientes portadores de doenças potencialmente fatais. Não basta ofertar conteúdo, é preciso avaliar a eficiência do ensino, ou seja, verificar se ele proporciona a aquisição das competências necessárias aos estudantes, que são definidas pelo conjunto de conhecimentos, habilidades e atitudes $2,3,7-10$.

Diante da pandemia da síndrome respiratória aguda grave do coronavírus 2 (severe acute respiratory syndrome coronavirus 2 - Sars-CoV-2), o ensino médico, desde 2020, precisou se adaptar ao ensino mediado por tecnologias (EMT), sem práticas ambulatoriais ou em enfermarias e com redução do contato informal entre estudante e estudante e entre eles e os docentes ${ }^{11}$. Assim, questionou-se como ocorreria a aquisição de competências nessa modalidade de ensino.

Experiências prévias de EMT mostraram que a educação em CP foi eficaz nas escolas médicas. As vantagens encontradas foram a flexibilidade do tempo de acesso do estudante e o controle sobre o ambiente de aprendizagem pelo professor. No entanto, impõe papel ativo ao estudante na busca pelo saber por meio da execução das tarefas e menor interação entre os atores. Ao professor compete o preparo das atividades de maneira extremamente planejada e intencional para o alcance dos objetivos de ensino propostos, com menor chance de adaptações em tempo real ${ }^{12-15}$.

Diante do contexto apresentado, este estudo teve como objetivo avaliar a aquisição de competências em $C P$, no nível da abordagem paliativa, entre estudantes de Medicina matriculados em uma disciplina de CP mediada por tecnologias.

\section{MÉTODO}

Este estudo obteve aprovação do Comitê de Ética em Pesquisa em Seres Humanos do Setor de Ciências da Saúde da Universidade Federal do Paraná (UFPR) e está registrado na Plataforma Brasil - Certificado de Apresentação para Apreciação Ética (CAAE) no 99340218.0.0000.0102. Todos os participantes foram orientados e assinaram o Termo de Consentimento Livre e Esclarecido. O estudo ocorreu de outubro a dezembro de 2020.

Todos os 45 estudantes de Medicina matriculados na disciplina de CP foram convidados para participar. A disciplina foi especificamente desenvolvida para o EMT, com carga horária de 40 horas, distribuídas ao longo de sete semanas. A ementa propunha atividades como aulas síncronas e assíncronas, leitura dirigida, problematização em casos clínicos e simulações.

O plano de ensino abordou introdução e conceito dos CP; manejo de sintomas desconfortáveis, como dor, respiratórios, digestivos e delirium; comunicação de más notícias; apoio psicossocial ao paciente e cuidador(es) não remunerados; 
abordagem da espiritualidade e anamnese espiritual; bioética em fim de vida e processo ativo de morte.

Os estudantes responderam individualmente ao instrumento de pesquisa PalliComp ${ }^{16}$ na semana que antecedeu o início das atividades da disciplina e, novamente, após a finalização das atividades e antes da divulgação das notas finais. O instrumento foi apresentado aos participantes por meio de formulário eletrônico Google Forms com preenchimento individual e anônimo. Pediu-se que não fossem consultados outros materiais durante o preenchimento.

O instrumento de pesquisa escolhido foi o PalliComp, que é um questionário validado para aferição de competências em $\mathrm{CP}$ entre estudantes de Medicina. Trata-se de um questionário de 25 itens na língua portuguesa, com as respostas dispostas em escala Likert de cinco pontos, e o participante assinala a alternativa mais adequada para cada afirmativa, com tempo de preenchimento estimado de 20 minutos $^{16}$.

Extraíram-se os dados obtidos em planilha eletrônica, e cada item recebeu a pontuação: +1 (totalmente correto), +0,5 (correto), 0 (alternativa neutra), $-0,5$ (incorreto) e -1 (totalmente incorreto). Atenção foi dada à correção de afirmativas propositalmente incorretas, que tiveram a pontuação invertida. A pontuação obtida foi agrupada em cada uma das dez competências e transformada em escores. Os escores foram calculados pela fórmula $=($ variável - nota mínima $) /($ nota máxima - nota mínima) e transformados numa escala 0 a 100. Compararam-se os escores antes e depois da intervenção.
Submeteram-se os dados à análise estatística pelo software Estatístico R, versão 3.6.3 (R Core Team). O nível de significância estatística adotado foi de 0,05. Aplicaram-se os testes de MannWhitney para amostras independentes.

\section{RESULTADOS}

Dos 45 estudantes matriculados na disciplina de CP, dois não puderam ser incluídos porque eram auxiliares de pesquisa, e 37 aceitaram participar da coleta de dados inicial (antes da disciplina) e 32 da final (após a disciplina).

A amostra de estudantes foi caraterizada por $68,9 \%$ de mulheres e $31,1 \%$ de homens, com idade média de 23,9 \pm 3,5 anos (mínimo 20 e máximo 37). Estavam matriculados no ciclo básico 12 estudantes (32,4\%) e 25 (67,6\%) no ciclo clínico. Apenas quatro alunos (10,8\%) participaram anteriormente de atividade extracurricular na área de $\mathrm{CP}$, com duração superior a seis meses, como liga acadêmica ou projeto de extensão.

O estudo das competências pode ser observado na Tabela 1 e Gráfico 1, com a comparação dos escores antes e depois da disciplina de CP. Exceto a competência 9 (comunicação), as restantes mostraram elevação dos escores antes e depois da disciplina. Entretanto, apenas as competências 1, 2, 3, 6 e 7 (respectivamente: conceito, manejo de sintomas físicos, psicoemocionais, família e bioética) apresentaram elevação estatisticamente significativa. Da mesma maneira, o escore geral de aquisição de competências mostrou elevação estatisticamente significativa.

Tabela 1. Desempenho médio dos estudantes, por escores, antes e depois da disciplina.

\begin{tabular}{|c|c|c|c|}
\hline \multirow{2}{*}{ Competências } & Estudo inicial $(n=37)$ & Estudo final $(n=32)$ & \multirow{2}{*}{ Valor de $p$} \\
\hline & Escore \pm desvio padrão & Escore \pm desvio padrão & \\
\hline Competência 1 & $61,1 \pm 29,0$ & $86,2 \pm 23,5$ & $<0,001$ \\
\hline Competência 2 & $46,7 \pm 22,3$ & $64,6 \pm 29,6$ & 0,004 \\
\hline Competência 3 & $58,1 \pm 24,3$ & $81,2 \pm 23,2$ & $<0,001$ \\
\hline Competência 4 & $75,7 \pm 26,8$ & $85,9 \pm 26,1$ & 0,07 \\
\hline Competência 5 & $64,9 \pm 24,6$ & $70,3 \pm 33,3$ & 0,13 \\
\hline Competência 6 & $79,0 \pm 23,2$ & $87,5 \pm 25,4$ & 0,03 \\
\hline Competência 7 & $66,9 \pm 27,7$ & $78,9 \pm 26,3$ & 0,05 \\
\hline Competência 8 & $52,7 \pm 28,7$ & $55,5 \pm 34,6$ & 0,67 \\
\hline Competência 9 & $55,2 \pm 23,1$ & $54,9 \pm 26,9$ & 1,00 \\
\hline Competência 10 & $79,3 \pm 29,8$ & $84,4 \pm 36,9$ & 0,13 \\
\hline Competência geral & $63,9 \pm 14,7$ & $74,9 \pm 14,6$ & 0,001 \\
\hline
\end{tabular}

Teste Mann-Whitney para amostras independentes; diferença estatisticamente significativa em negrito.

Competência 1. Aplicar os constituintes centrais dos CP no ambiente próprio e mais seguro para os doentes e as famílias; Competência 2. Aumentar o conforto físico durante as trajetórias de doença dos doentes; Competência 3. Atender às necessidades psicológicas dos doentes; Competência 4. Atender às necessidades sociais dos doentes; Competência 5. Atender às necessidades espirituais dos doentes; Competência 6. Responder às necessidades dos cuidadores familiares em relação aos objetivos do cuidar em curto, médio e longo prazos; Competência 7. Responder aos desafios da tomada de decisão clínica e ética em CP; Competência 8. Implementar uma coordenação integral do cuidar e um trabalho de equipe interdisciplinar em todos os contextos em que os CP são oferecidos; Competência 9. Desenvolver competências interpessoais e comunicacionais adequadas aos CP; Competência 10. Promover o autoconhecimento e o contínuo desenvolvimento profissional. 
Gráfico 1. Desempenho médio dos estudantes.
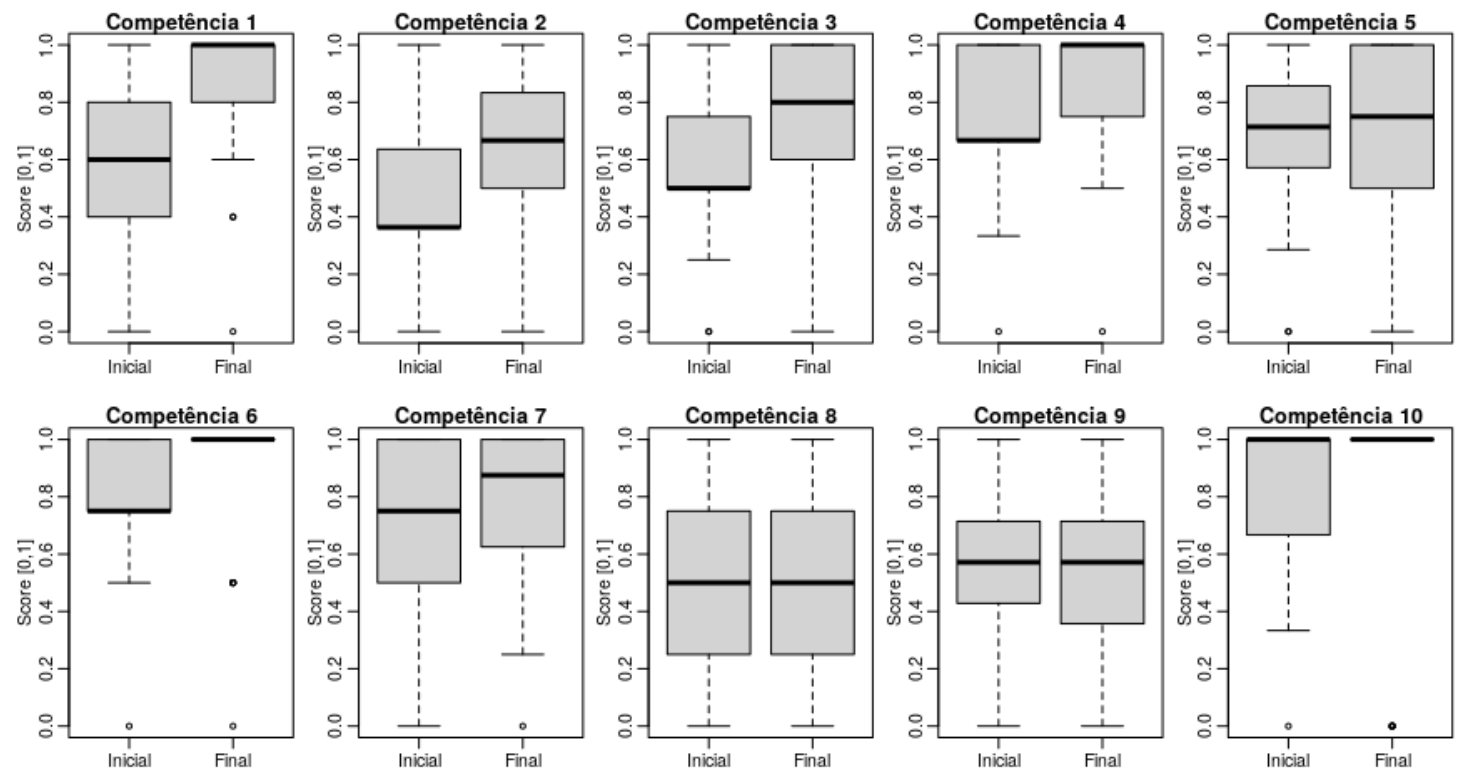

Teste Mann-Whitney para amostras independentes; foi observada diferença estatisticamente significativa nas competências 1, 2, 3, 6 e 7.

Competência 1. Aplicar os constituintes centrais dos CP no ambiente próprio e mais seguro para os doentes e as famílias; Competência 2. Aumentar o conforto físico durante as trajetórias de doença dos doentes; Competência 3. Atender às necessidades psicológicas dos doentes; Competência 4. Atender às necessidades sociais dos doentes; Competência 5. Atender às necessidades espirituais dos doentes; Competência 6. Responder às necessidades dos cuidadores familiares em relação aos objetivos do cuidar em curto, médio e longo prazos; Competência 7. Responder aos desafios da tomada de decisão clínica e ética em CP; Competência 8. Implementar uma coordenação integral do cuidar e um trabalho de equipe interdisciplinar em todos os contextos em que os CP são oferecidos; Competência 9. Desenvolver competências interpessoais e comunicacionais adequadas aos CP; Competência 10. Promover o autoconhecimento e o contínuo desenvolvimento profissional.

\section{DISCUSSÃO}

A pandemia da Sars-Cov-2 trouxe desafios superlativos à sociedade em geral e à saúde pública na maior parte do mundo. A educação médica, que consistia em atividades essencialmente presenciais, enfrentou desafios para se adaptar. No curso avaliado, as atividades tiveram que ser rapidamente adaptadas ao EMT e sem a certeza do sucesso da aquisição de competências educacionais. Os estudantes, apesar de pertencerem à geração nativa digital, não tinham familiaridade com a plataforma de ensino adotada pela universidade e o ensino regular nessa modalidade.

É importante ressaltar que o conceito de competências utilizado neste estudo foi aquele definido por Perrenoud et al. ${ }^{10}$ em 2002, que consiste na capacidade de o "educando agir eficazmente em um determinado tipo de situação, apoiado pelos conhecimentos, mas sem limitar-se a eles" (p.141). Segundo os autores, a escola, de fato, encarrega-se de transferir conhecimentos, mas nem sempre é capaz de ligar esses saberes com a própria vida do estudante ou com a realidade profissional. Assim, o estudo pode ficar dissociado da realidade. Recomenda-se que a escola trabalhe para a aquisição de proficiência, ou seja, que o estudante domine as habilidades necessárias para o desempenho de uma determinada profissão e seja capaz de agir eticamente em direção às atitudes profissionais desejáveis ${ }^{2,3,9,10}$.
Este estudo demonstrou que a estratégia adotada para o ensino foi razoavelmente bem-sucedida, uma vez que a competência geral dos estudantes se elevou de maneira significativa, mas que poderá ser aprimorada para que todas as competências apresentem melhores escores no futuro. Possivelmente, as atividades práticas no ambiente assistencial poderiam estimular os estudantes especialmente naquelas competências em que o aprendizado se dá com a instrumentalização seguida da interação humana, como a comunicação e o trabalho em equipe.

Os discentes adquiriram familiaridade com a definição e os princípios dos $\mathrm{CP}$, e a abordagem de sintomas físicos desconfortáveis e do sofrimento psicoemocional, o que pode ser demonstrado pela elevação significativa dos escores nas competências 1, 2 e 3. Trata-se de saberes importantes para a prática assistencial do futuro médico generalista, mas não são suficientes de maneira isolada. A abordagem dos sofrimentos social e espiritual também faz parte das competências essenciais voltadas para a saúde integral de pessoas adoecidas, contudo, por falta de preparo dos profissionais de saúde, raramente é inserida nos cuidados ${ }^{17,18}$. Infelizmente, a elevação observada nos escores das competências 4 e 5 foi pequena e não apresentou diferença estatisticamente significativa, o que indica a necessidade de revisão da estratégia de ensino para essas competências. 
A aquisição de competências para abordar os familiares dos pacientes e apoiar o planejamento dos cuidados e a tomada de decisão ética em CP mostraram elevação significativa dos escores nas competências 6 e 7. No entanto, não houve elevação dos escores de competências relacionadas com o trabalho em equipe, comunicação e autodesenvolvimento (competências 8, 9 e 10), que são competências adquiridas essencialmente na prática, com interação entre os estudantes, os pacientes e a supervisão docente.

Neste estudo, a competência da comunicação, infelizmente, mostrou pequena redução dos escores, sem diferença estatística significativa, apesar de a temática ter sido abordada na disciplina de maneira teórica, seguida de prática, discussão em equipe e debriefing. OEMT proporcionou o contato dos estudantes com a temática, mas possivelmente não foi capaz de permitir, de fato, o treinamento desejável sobre os CP. A aquisição de competências ocorre gradualmente, de acordo com a exposição do estudante aos desafios e às necessidades impostas no processo de ensino ${ }^{2,3,9}$, e a obrigatoriedade do distanciamento físico provocada pela pandemia, apesar de necessária, resultou em algum prejuízo ao ensino médico ${ }^{11}$.

Pode-se supor que o EMT tenha sido frágil para o desenvolvimento da segurança entre os estudantes em habilidades práticas desafiadoras, nas quais o processo de aprendizado leva tempo e ocorre por meio das próprias experiências. É provável que, num primeiro momento, o estudante tenha se conscientizado do que não sabia, ou seja, tenha passado do estágio da incompetência inconsciente para a incompetência consciente e possivelmente para a competência consciente. Todavia, o aprendizado se mostrou superficial e frágil. Apesar do contato com a oportunidade do aprendizado, é compreensível que o discente fique inseguro em avaliações precoces ${ }^{19,20}$.

A pesquisa de satisfação dos estudantes com a modalidade de ensino consistiu em uma roda de conversa por videoconferência e feedback voluntário no final do curso, e, infelizmente, não foi quantificada. A maioria relatou satisfação, mas alguns apontaram que o método foi exigente e desafiador, e os alunos não estavam seguros do aprendizado, pois tratava-se da primeira experiência de aprendizado on-line. Estudantes de medicina norte-americanos e alemães obtivem ganhos equivalentes de conhecimento com o EMT e o ensino tradicional, entretanto aqueles mostraram menor satisfação no EMT $^{13}$ e estes apresentaram maior satisfação ${ }^{15}$.

A satisfação é uma experiência subjetiva e envolve variáveis que nem sempre podem ser aferidas ou controladas. A discrepância da satisfação com relação ao EMT pode ser atribuída, entre outros fatores, à dificuldade de adaptação às metodologias on-line e ao grau de experiência tanto de docentes como de discentes ${ }^{12,15}$. Esse fato deve ser levado em consideração apesar da falta de opções de ensino durante a pandemia. Os estudantes e os docentes tiveram que aprender e se adequar ao método de ensino, apesar da ausência de experiência prévia em EMT na universidade avaliada.

A metodologia inserida no EMT inclui diversas formas de ensino anteriormente empregadas no ensino presencial. A ementa didática da disciplina avaliada neste estudo foi composta com as temáticas essenciais dos $\mathrm{CP}$, como introdução e conceito dos CP; manejo de sintomas desconfortáveis (por exemplo: dor, dispneia, constipação e delirium); comunicação de más notícias; apoio psicossocial ao paciente e cuidador(es) não remunerados; abordagem da espiritualidade e anamnese espiritual; bioética em fim de vida e processo ativo de morte. A mesma temática era ofertada no ensino presencial anterior à pandemia, mas que, curiosamente, não havia sido qualificada.

Não é possível separar totalmente as competências avaliadas neste estudo, visto que sua aquisição ocorre de maneira simultânea em várias áreas do saber ${ }^{10}$. De maneira geral, para as questões técnicas optou-se por aulas expositivas, leitura dirigida e problematização por meio de casos clínicos. Para as questões humanas, optou-se por discussão em grupo e simulação, com debriefing. E para as questões relacionais, tentou-se a resolução de tarefas em equipe, após reflexão individual.

O que se pode observar é que as competências essencialmente objetivas e claramente relacionadas com o trabalho do médico, como o conceito de CP e o manejo de sintomas, apresentaram notável aquisição de competências. Em relação às competências subjetivas e, talvez, mais distantes da percepção estudantil de que fazem parte do trabalho convencional do médico - como a abordagem social e espiritual -, não mostraram elevação significativa dos escores. Ou seja, a aquisição de competências objetivas foi notável, o que não ocorreu com as subjetivas. Já as competências relacionais, com o trabalho em equipe e a comunicação, apesar de abordadas, não mostraram ganhos.

A complexidade do ensino médico nos CP foi abordada por Boland et al. ${ }^{12}$. Ensino, vivência e treinamento são imprescindíveis para que o futuro médico compreenda as necessidades assistenciais dos pacientes em fim de vida. $\mathrm{O}$ EMT poderá ofertar conhecimentos e aquisição de algumas habilidades, mas possivelmente não substituirá o contato humano e o aprendizado a ele relacionado. Estudantes turcos que foram submetidos ao ensino de CP por EMT também mostraram aumento de competências em relação aos controles ${ }^{14}$. Pode-se compreender que ofertar EMT é uma estratégia educacional válida e que merece atenção nos cursos de Medicina, mas possivelmente é essencial incluir práticas ambulatoriais e hospitalares para formar futuros médicos 
competentes nas questões do fim de vida. Também é possível que o amadurecimento das estratégias educacionais, após esse o período inicial de experimentação docente e discente, mostre resultados mais favoráveis.

Apesar de diversas competências fundamentais aos CP estarem implícitas no ensino médico, a subjetividade necessária à humanização está fortemente vinculada à interação humana. Em estudos que avaliaram o desenvolvimento específico da comunicação com alunos de Medicina, verificou-se, de maneira geral, a exigência de maior carga horária de treinamento prático, de vivência e de reflexão em grupo ${ }^{21}$. Ainda, os estudantes podem subestimar a própria capacidade ou superestimá-la de acordo o grau de confiança depositado na experiência de ensino, o que não necessariamente está relacionado com a aquisição real de competências ${ }^{14,21,22}$.

A relação da subjetividade na aquisição de competências também esteve presente em outros estudos. Em 2017, Hagiwara et al. ${ }^{23}$ observaram que a dificuldade de abordagem de questões espirituais e religiosas estava relacionada com diferenças culturais e a diversidade de interpretações entre profissionais de saúde e pacientes. Em 2014, Anneser et al. ${ }^{24}$ perceberam que a experiência pessoal do estudante tem influência sobre a aprendizagem. As emoções mal elaboradas ou não reconhecidas sobre o pesar e a morte podem interferir na capacidade dos futuros médicos de lidar de forma adequada com os medos e as necessidades dos pacientes e familiares no final da vida. Sendo assim, cada estudante é singular, traz uma bagagem de experiências, e esses fatores tem influência sobre a percepção dos $C P$, assim como na aquisição de competências.

Ainda, vale ressaltar que todas as competências listadas estão fortemente entrelaçadas. Descrevê-las de maneira separada é uma facilitação didática, mas que não corresponde ao mundo real. A comunicação qualificada é essencial para a interação médico-paciente, médico-família e médico-equipe, e aumenta a possibilidade de o profissional escutar pessoas com empatia, dividir informações sobre terapêuticas, diagnóstico e prognóstico, trabalhar em equipe, acolher familiares e ter decisões éticas ${ }^{3,23-26}$.

O aprendizado referente à comunicação e ao trabalho em equipe é essencial ao futuro médico generalista que se propõe ao atendimento humanizado, especialmente quando se trata de pessoas na fase final de vida e vulneráveis. O distanciamento físico imposto pela pandemia pode ter sido um dificultador para a melhora dos escores dessas competências, mas tal condição poderá resultar em um ensino mais bem estruturado no futuro diante das necessidades atuais.

Reconhecer o que se sabe e o que carece de aprimoramento é parte do desenvolvimento profissional, e, infelizmente, a amostra avaliada ainda não adquiriu a competência de maneira satisfatória, demonstrada pela competência 10. O profissionalismo refere-se ao potencial do indivíduo para realizar o trabalho de forma competente e plausível, desenvolver práticas médicas seguras, compreender os limites das próprias habilidades e agir motivado pelos melhores interesses dos pacientes ${ }^{2,3,27,28}$.

Como limitação ressalta-se que a disciplina ofertada foi o primeiro contato formal em CP da maioria dos estudantes avaliados. O sucesso na aquisição de competências era desejado, e houve esforço no desenho da estratégia de ensino, mas não se pode atribuir grande expectativa a uma disciplina no seu primeiro ciclo de oferta mediado por tecnologia e sem práticas assistenciais. Há necessidade de mais estudos para se concluir de maneira definitiva a melhor forma de ensinar CP no curso de Medicina. Felizmente, os estudantes vivenciarão outros momentos de aprendizado nos estágios práticos.

O EMT possui fragilidades, assim como o ensino anteriormente desenvolvido. É necessário avaliar a aquisição de competências independentemente da metodologia ofertada, sobretudo em temáticas emergentes que não têm ementa didática predefinida. O EMT não pode ser reduzido a aulas por videoconferências síncronas ou assíncronas, mas deveria agregar a tecnologia existente para levar o estudante à formação desejável ao médico generalista e definida nas Diretrizes Curriculares Nacionais ${ }^{29}$.

$\mathrm{Na}$ pandemia, professores e estudantes precisaram adquirir competências tecnológicas de ensino e aprendizagem. Interesse, estudo, dedicação, reavaliação dos métodos de ensino e aferição da aquisição de competências representaram o começo desse processo. Não se deveria reduzir o estudo da aquisição de competências apenas ao período da pandemia, uma vez que as ferramentas de ensino incorporadas são atemporais. O EMT traz um universo de novas perspectivas pouco utilizadas no ensino médico brasileiro, como a possibilidade do ensino híbrido, a gamificação, entre outros.

Os resultados deste estudo permitem compreender que o ensino ofertado permitiu aquisição parcial das competências em CP no nível da abordagem paliativa entre os estudantes de Medicina avaliados. O desempenho foi satisfatório nas competências relacionadas com o conceito de $C P$, as abordagens física e emocional, a família e a tomada de decisão ética. Não houve desempenho satisfatório nas competências relacionadas com as abordagens social e espiritual, o trabalho em equipe, a comunicação e o autodesenvolvimento.

\section{CONCLUSÃO}

Em plena pandemia, ensinar CP aos estudantes de Medicina foi um processo desafiador. Por conta da suspensão das atividades presenciais, houve a necessidade de adequar o 
ensino ao EMT, sem experiência tecnológica anterior tanto dos docentes como dos discentes. A estratégia de ensino ofertada mostrou que o escore geral de competências em CP aumentou de maneira significativa. Conclui-se que houve aquisição de competências em CP no nível da abordagem paliativa com EMT entre estudantes de Medicina e que a estratégia de ensino se mostrou válida, porém, a partir deste estudo, deve-se revisar a proposta didática para direcionar o aprendizado a cada uma das competências desejáveis aos futuros médicos.

\section{AGRADECIMENTOS}

As autoras agradecem aos estudantes participantes da pesquisa.

\section{CONTRIBUIÇÃO DAS AUTORAS}

Amanda Carla de Souza Kanashiro e Rebeca Isabela Ciardulo Marques Grandini participaram da coleta e organização dos dados, e da redação e revisão do texto. Úrsula Bueno do Prado Guirro idealizou a pesquisa, participou da aprovação ética, da organização dos dados e da redação e revisão do texto, e orientou o estudo.

\section{CONFLITO DE INTERESSES}

Declaramos não haver conflito de interesses.

\section{FINANCIAMENTO}

\section{Declaramos não haver financiamento.}

\section{REFERÊNCIAS}

1. World Health Organization. Palliative care. WHO, 2020 [acesso em 5 jun 2021]. Disponível em: https://www.who.int/news-room/fact-sheets/detail/ palliative-care.

2. Gamondi C, Larkin P, Payne S. Core competencies in palliative care: an EAPC white paper on palliative care education: part 1. Eur J Pall Care. 2013 Mar;20(2):86-91.

3. Gamondi C, Larkin P, Payne S. Core competencies in palliative care: an EAPC white paper on palliative care education: part 2. Eur J Pall Care. 2013 May;20(3):140-5.

4. Linklater GT, Bowden J, Pope L, McFatter F, Hutchison SMW, Carragher PJ, et al. Developing learning outcomes for medical students and foundation doctors in palliative care: a national consensus-seeking initiative in Scotland. Med Teach. 2014;36(5):441-6.

5. Academia Nacional de Cuidados Paliativos. Panorama dos cuidados paliativos no Brasil. São Paulo: ANCP; 2018 [acesso em 28 abr. 2021]. Disponível em: https://paliativo.org.br/wpcontent/uploads/2018/10/ Panorama-dos-Cuidados-Paliativos-no-Brasil-2018.pdf.

6. Santos AFJ, Ferreira EAL, Guirro UBP. Atlas dos cuidados paliativos no Brasil 2019. São Paulo: ANCP; 2020 [acesso em 01 set 2021]. Disponível em: https://api-wordpress.paliativo.org.br/wp-content/uploads/2020/05/ ATLAS_2019_final_compressed.pdf.

7. Frey RA, Gott M, Neil H. Instruments used to measure the effectiveness of palliative care education initiatives at the undergraduate level: a critical literature review. BMJ Support Palliat Care. 2013;3(1):114-9. doi: 10.1136/ bmjspcare-2012-000306.
8. Lehto JT, Hakkarainen $\mathrm{K}$, Kellokumpu-Lehtinen PL, Saarto $\mathrm{T}$. Undergraduate curriculum in palliative medicine at Tampere University increases students' knowledge. BMC Palliat Care. 2017;16(1):13. doi: 10.1186/s12904-016-0182-8.

9. Scallon G. Avaliação da aprendizagem numa abordagem por competências. Curitiba: PUCPRESS; 2015.

10. Perrenoud $P$, Thurler MG, Macedo L, Machado NJ, Allessandrini CD. As competências para ensinar no século XXI: a formação de professores e o desafio da avaliação. Porto Alegre: Penso; 2002.

11. Yusoff MSB, Hadie SNH, Mohamad I, Draman N, Muhd Al-Aarifin I, Wan Abdul Rahman WF, et al. Sustainable medical teaching and learning during the Covid-19 pandemic: surviving the new normal. Malays J Med Sci. 2020;27(3):137-42.

12. Boland JW, Brown MEL, Duenas A, Finn GM, Gibbins J. How effective is undergraduate palliative care teaching for medical students? A systematic literature review. BMJ Open. 2020;10(9):e036458.

13. Day FC, Srinivasan M, Der-Martirosian C, Griffin E, Hoffman JR, Wilkes MS. A comparison of Web-based and small-group palliative and end-of-life care curricula: a quasi-randomized controlled study at one institution. Acad Med. 2015;90(3):331-7. doi: 10.1097/ACM.0000000000000607.

14. Kudubes AA, Bektas M. The effect of web-based pediatric palliative care education on the palliative care knowledge level and practices of nursing students. Perspect Psychiatr Care. 2020;56(3):533-40.

15. Schulz-Quach C, Wenzel-Meyburg U, Fetz K. Can elearning be used to teach palliative care? Medical students' acceptance, knowledge, and selfestimation of competence in palliative care after elearning. BMC Med Educ. 2018;18(1):82. doi: 10.1186/s12909-018-1186-2.

16. Guirro UBP, Perini CC, Siqueira JE. PalliComp: um instrumento para avaliar a aquisição de competências em cuidados paliativos. Rev Bras Educ Med. 2021;45(3):e140.

17. Puchalski CM, King SDW, Ferrell BR. Spiritual considerations. Hematol Oncol Clin North Am. 2018 June;32(3):505-17. doi: 10.1016/j.hoc.2018.01.011.

18. Koper I, Pasman HRW, Schweitzer BPM, Kuin A, Onwuteaka-Philipsen BD. Spiritual care at the end of life in the primary care setting: experiences from spiritual caregivers - a mixed methods study. BMC Palliat Care. 2019;18(1):98. doi: 10.1186/s12904-019-0484-8.

19. LaDonna KA, Ginsburg S, Watling C. "Rising to the level of your incompetence": what physicians' self-assessment of their performance reveals about the imposter syndrome in medicine. Acad Med. 2018 May;93(5):763-8. doi: 10.1097/ACM.0000000000002046.

20. LaDonna KA, Ginsburg S, Watling C. Shifting and sharing: academic physicians' strategies for navigating underperformance and failure. Acad Med. 2018 Nov;93(11):1713-8. doi: 10.1097/ACM.00000000000002292.

21. Storarri ACM, de Castro GD, Castiglioni L, Cury PM. Confidence in palliative care issues by medical students and internal medicine residents. BMJ Support Palliat Care. 2019 Mar;9(1):e1. doi: 10.1136/ bmjspcare-2017-001341.

22. Cowfer B, McGrath C, Trowbridge A. Teaching pediatric palliative care communication skills to fourth-year medical students through roleplay. MedEdPORTAL. 2020 Oct 16;16:10991. doi: 10.15766/mep_23748265.10991.

23. Hagiwara $Y$, Ross J, Lee $S$, Sanchez-Reilly $S$. Tough conversations: development of a curriculum for medical students to lead family meetings. Am J Hosp Palliat Care. 2017;34(10):907-11. doi: 10.1177/1049909116669783.

24. Anneser J, Kunath N, Krautheim V, Borasio GD. Needs, expectations, and concerns of medical students regarding end-of-life issues before the introduction of a mandatory undergraduate palliative care curriculum. $J$ Palliat Med. 2014;17(11):1201-5. doi: 10.1089/jpm.2013.0614.

25. You JJ, Downar J, Fowler RA, Lamontagne F, Ma IW, Jayaraman D, et al. Barriers to goals of care discussions with seriously ill hospitalized patients and their families: a multicenter survey of clinicians. JAMA Intern Med. 2015 Apr;175(4):549-56. Errata: JAMA Intern Med. 2015 Apr;175(4):659. 
26. Morgan DD, Litster C, Winsall M, Devery K, Rawlings D. "It's given me confidence": a pragmatic qualitative evaluation exploring the perceived benefits of online end-of-life education on clinical care. BMC Palliat Care. 2021;20(1):57. doi: 10.1186/s12904-021-00753-y.

27. Stockley AJ, Forbes K. Medical professionalism in the formal curriculum: 5th year medical students' experiences. BMC Med Educ. 2014;14:259. doi: 10.1186/s12909-014-0259-0.
28. SaadTC, Riley S, Hain R. A medical curriculum in transition: audit and student perspective of undergraduate teaching of ethics and professionalism. J Med Ethics. 2017;43(11):766-70. doi: 10.1136/medethics-2016-103488.

29. Brasil. Diretrizes Curriculares Nacionais do Curso de Graduação em Medicina. Brasília: Ministério da Educação; 2014 [acesso em 5 jun 2021]. Disponível em: http://portal.mec.gov.br/index.php?option=com docman\&view=download\&alias=15514-pces116-14\&category slug=abril-2014-pdf\&ltemid=30192. 